Mit breiter Zustimmung kann heutzutage rechnen, wer die These vertritt, trotz fortbestehender sozialer Ungleichheit hätten sich die Lebenschancen der Menschen in den wohlfahrtsstaatlichen Massendemokratien so grundlegend geändert, daß das Hierarchiemodell sozialer Klassen und Schichten seinen Realitätsgehalt einbüßt. Dies hat, unter Hinweis auf Erosionsprozesse subkultureller Klassenideologien und ständisch eingefärbter Klassenlagen sowie mit dem Verweis auf Prozesse zunehmender Individualisierung von Lebenslagen, U. Beck vor gar nicht so langer Zeit mit seiner provozierenden Behauptung getan: In der Bundesrepublik etabliere sich gegenwärtig eine "Einheitsgesellschaft der Unselbständigen «, in der, jenseits sozialer Klassendifferenzierung, die Homogenisierung individualisierter, isolierter und familialer Privatexistenzen zunehmend an Bedeutung gewinnt. Parallel zu diesem Prozeß, so Beck weiter, entstehen neue soziale Formationen und neue soziale Identitäten, werden neue Ideologien hervorgetrieben und können sich neuartige Koalitionen und Konfliktlinien herauskristallisieren, die die gewohnten Polarisierungen der traditionellen Klassenzugehörigkeit unterlaufen.

Wenn eine solche Einschätzung den Zeitgeist halbwegs richtig trifft, so ist es in keiner Weise selbstverständlich, ein Schwerpunktheft zum Thema »Klassen und Herrschaft « vorzulegen. Wie schon bei der letzten Nummer über aktuelle Auseinandersetzungen der marxistischen Krisentheorie (PROKLA 57) scheint ein derart *anti-zyklisches « Publikationsgebaren, in der bundesrepublikanischen Diskussionslandschaft zumindest, nach besonderer Rechtfertigung zu verlangen: Im Gegensatz zum angelsächsischen Raum, wo Bemühungen um eine "revidierte Klassentheorie«: auch in den letzten Jahren nicht eingestellt wurden, ist die Situation hierzulande eher dadurch gekennzeichnet, daß die Frage des Zusammenhangs von ökonomischer Theorie und einer Theorie des gesellschaftlichen Konflikts keine große Aufmerksamkeit mehr erweckt. - Ein nur flüchtiger Blick in die zeitgenössische Publizistik mag dabei den Eindruck erwecken, als sei die marxistische Klassentheorie, nicht zuletzt wegen ihrer Privilegierung der »objektiven « Klasseninteressen des Proletariats in der Bundesrepublik vor allem deswegen so uninteressant geworden, weil hier die von "non-class-issues" zusammengehaltenen neuen sozialen Bewegungen eine so überdurchschnittlich große Verbreitung gefunden haben. Ohne die theoretischen Sackgassen, in die die westdeutsche Linke in den.70er Jahren mit Verbissenheit und geradezu kontrafaktischem Optimismus hineingestürmt war, läßt sich das aktuelle Interesse an den »neuen sozialen Identitäten « und an klassenjenseitigen Konfliktlinien indes nicht hinreichend erklären. Ihr auf polit-ökonomische Fragen verengter Marxismus verhinderte mit einigem Erfolg, das Eigengewicht, welches politischen und rechtlichen Phänomenen und Institutionen für die Ausdifferenzierung moderner Sozialverhältnisse zukommt, überhaupt zur Kenntnis zu nehmen; er blockierte notwendige Einsichten in kulturelle, ethnische, religiöse oder nationale Eigenheiten, die quer und unterhalb von ökonomisch definierten Klassenlagen liegen, aber die Lebenschancen und die politischen und moralischen Orientierungen sozialer Akteure wesentlich beeinflussen kön- 
nen. Politik, Recht und Alltagskultur erschienen folgerichtig als lediglich »abgeleitete Sphären « ökonomisch begründeter gesellschaftlicher Interessen; und sofern die Handlungen sozialer Akteure, insbesondere aber die der »alten « oder der »neuen « Arbeiterklasse, von Einflüssen dieser Sphären "bürgerlicher Ideologie«stärker geleitet schienen als von der spezifischen Rationalität ihrer »objektiven Klassenlage «, so war von "falschem Bewußtsein « oder von der übermächtigen Herrschaft des Warenfetischismus die Rede. Jene krud mechanistischen Modelle des Zusammenhangs von sozioökonomischer Struktur und sozialem Handeln, die dem Anspruch gerechtzuwerden versuchten, eine Analyse kapitalistischer Produktions- und Herrschaftsverhältnisse und zugleich eine Theorie politischer und sozialer Transformationsprozesse zu liefern, dienten zum einen dazu, das augenscheinliche Prognosedefizit der marxistischen Klassenbewußtseinstheorie zu verdecken; sie eignete sich aber zum anderen auch dafiir, an der Idee eines der Arbeiterklasse eigenen und sie damit von allen anderen Sozialgruppierungen unterscheidenden »objektiven Interesses« an der Herstellung sozialistischer Gesellschaftsverhältnisse festhalten zu können. D. Lockwood erinnert in seinem in diesem Heft abgedruckten Beitrag nicht zu Unrecht daran, daß derartige Bemühungen, man denke nur an die "Ideologischen Staatsapparate « der strukturalistischen Schule um L. Althusser, am Ende die funktionalistischen Prämissen der »bürgerlichen Soziologie eines T. Parsons von links nachvollzogen.

In der Bundesrepublik, aber eben nicht nur hier, hat sich diese Tradition objektivistischer Erklärungen von sozialem Bewußtsein und kollektivem Handeln in zähen Disputen über die Bedeutung der Unterschiede zwischen produktiver und unproduktiver Arbeit niedergeschlagen. Sie prägte den Ableitungs-Marxismus und die vielfältigen Bemühungen, eine »neue (technisch-wissenschaftliche) Arbeiterklasse« als Bündnispartner der alten, vom "sozialdemokratischen Klassenkompromiß « aber leider hoffnungslos korrumpierten Arbeiterklasse zu identifizieren.

Gemeinsam war all diesen Bestrebungen, daß sie - vor dem Hintergrund einer seit den 20er Jahren anhaltenden Zunahme von Angestellten gegenüber Arbeitern - eine direkte Verbindung zwischen der ökonomischen Theorie der Systemwidersprüche und der politischen Soziologie des Klassenkonflikts herzustellen versuchten. Und dies ist auch ein Motiv der neueren Versuche geblieben, Probleme, die der Klassentheorie von Seiten der mneuen Mittelklasse « her erwachsen, theoretisch in den Griff zu bekommen. So bemüht sich beispielsweise $E$.O. Wright seit kurzem, der Klassentheorie dadurch zu neuer Aktualität zu verhelfen, daß er, in Anlehnung an J. Roemer, eine Ausweitung und eine interne Differenzierung des Ausbeutungsbegriffs vornimmt, um so die - im Hinblick auf die »neue Mittelklasse« ja höchst erklärungsschwache - Werttheorie umgehen zu können. C. Offe hat in seinem Kommentar zu Wrights spieltheoretisch fundiertem Ausbeutungsbegriff, nach dem alle kollektiven Akteure typischerweise in Nutzenmaximierungsaktivitäten engagiert und in eine Pluralität von Ausbeutungsverhältnissen verstrickt sind, aber völlig zu recht klargestellt: Daß durch die Beiseitelassung aller Phänomene sozialer Herrschaft und durch die Konzentration auf resp. die Ausweitung des Ausbeutungsbegriffs dieser seine kritische Potenz vor allem deswegen verlieren muß, "weil die Zentralität der Ausbeutungsbeziehung zwischen Lohnarbeit und Kapital, die in der bürgerlichen Gesellschaft zugleich rechtlich privilegiert und rechtlich dementiert wird, aus dem Blick gerät«。

Was diese neueren Rettungsversuche der Klassentheorie aber schließlich auch noch mit der älteren Tradition strukturtheoretisch akzentuierter Marxismusrezeption verbindet, ist ihre Fixierung auf ein Konzept sozialen Handelns, das der vom klassischen Utilitarismus favorisierten Vorstellung "instrumenteller Rationalität« recht eng verpflichtet bleibt. Auch dort, 
wo, wie beispielsweise bei J. Elster, einem der prominentesten Vertreter des spieltheoretischen Marxismus, die Vorstellung aufgegeben ist, daß die Klassenstruktur in allen Gesellschaften die Haupterklärung der sozialen Konflikte zwischen organisierten Gruppen darstellt, und wo der machtgenerierenden Rolle politischer Institutionen wie der Konfliktrelevanz kultureller Identitäten ein größeres theoretisches Gewicht eingeräumt wird, bleibt jedes utilitaristische Handlungskonzept zentral. Unbestritten ist sicher, und darin kann man mit C. Offe die Leistung von Elsters Diskussionsbeitrag sehen, daß hier die konstitutive Rolledes Staates für Prozesse der Klassenbildung eher wahrgenommen wird als in jenen Varianten der marxistischen Klassentheorie, die die politischen Rechtsbeziehungen im Kapitalismus als ökonomisch determinierte Phänomene »bürgerlicher Ideologie« abhandeln. - Aus einer ganz anderen Perspektive, nämlich der einer »links-weberianisch « inspirierten Sozialstaatsanalyse heraus, zeigt M. Krätke in seinem Beitrag, daß es in der Tat selbst in vollentwickelten bürgerlichen Gesellschaften institutionalisierte Ungleichheiten gibt, die nicht (allein) auf der Ungleichheit zwischen gesellschaftlichen Klassen beruhen, sondern auf askriptiven Diskriminierungen, »die die 'Klasseneinheit' der Lohnarbeiter im Wohlfahrtsstaat einstweilen illusorisch erscheinen lassen « und die insbesondere besitzlose Nicht-Lohnarbeiter zu Sozialstaatsbürgern »minderen Rechts« stempeln.

Fraglich ist indes, wie solch notwendige Korrekturen an dem monokausalen Erklärungsmodell der Klassentheorie zu einer komplexen und zugleich richt-funktionalistischen Sozialtheorie hinführen könnten, wenn deren utilitaristisches Handlungskonzept beibehalten wird. Ch. Taylors Einwand, der spieltheoretische Marxismus, der sich das soziale Leben ja als Spiel rational gegeneinander handelnder Individuen vorstellt, könne Gültigkeit eigentlich nur für atomisierte Gesellschaften beanspruchen, für Sozialgebilde also, in denen keine Normen von "Sittlichkeit« resp. gemeinsam geteilte Werte und Normen Geltung haben, deckt sich in etwa mit dem von D. Lockwood immer wieder erhobenen Vorwurf: Alle Phänomene, die die »bürgerliche Soziologie « als zentrale Elemente von konsensgetragener Sozialintegration so scharf akzentuiert, entgingen der marxistischen Klassentheorie deswegen, weil ihr kognitivistisch verengtes Ideologieverständnis sie stets daran gehindert habe, den normativen und moralischen Bestandteilen der Handlungsorientierungen sozialer Akteure nachzuspüren.

Notwendig scheint darum wohl zuallererst eine kategoriale Erweiterung der Klassentheorie um eben solche normativen Elemente eines strikt nicht-utilitaristischen Handlungskonzepts. - Vorerst, soviel läßt sich aus den vorliegenden, hier leider nur in einer schmalen Auswahl vorstellbaren Beiträgen zum Thema entnehmen, ist wohl davon auszugehen, daß: 1. mit sozialen Formationen und Identitäten gerechnet werden muß, die sowohl unterhalb von ökonomisch beschreibbaren Klassenlagen positioniert sind als diese auch durchkreuzen können; 2. systembedrohende Konflikte denkbar geworden sind, die nicht allein in der Polarität von Kapital und Lohnarbeit verankert sind; 3. eindeutige Zuordnungen von sozialen Klasseninteressen und bestimmten Typen politischer Optionen der möglichst präzisen Analyse ökonomischer Klassenlagen nicht mehr erwartet werden dürfen.

Auf welche Weise veränderte ökonomische Strukturbedingungen auch die Form von Konflikten beeinflussen können, die eindeutig um die Achse Lohnarbeit und Kapital gelagert sind, zeigen Michael Burawoy und Anne Smith. Anhand vier sorgfältig ausgewählter Fallstudien zu Betriebsstillegungen und -verlagerungen wird die These einer heraufziehenden "hegemonialen Despotie « entwickelt, die entweder traditionelle Widerstandsformen der Betroffenen ins Leere laufen läßt oder die Betroffenen direkt partikularisiert und individualisiert, und 
auf diese Weise Konflikte absorbiert. Eine Entwicklungstendenz, die nicht allein in der USIndustrie zu beobachten ist.

Mit dem Beitrag von Alain Lipietz schließlich, setzen wir die im letzten Heft begonnene Diskussion zur Krisentheorie fort. Lipietz gehört zusammen mit Michel Aglietta und Robert Boyer zu den prominentesten Vertretern der französischen "Regulationsschule«, die sich darum bemüht, von den Grundlagen der Marxschen Kritik der Politischen Ökonomie aus eine krisentheoretische Sichtweise fortzuentwickeln, die auch die entwickeltsten Erscheinungen des Krisenprozesses erfassen kann. Wir haben diesen Aufsatz ausgewählt, weil er eine gute Einführung in ein hierzulande wenig bekanntes Theoriekonzept darstellt (und weil wir hoffen, so die schlechte Tradition einer vorschnellen, nur auf die Schlüsselbegriffe zielende Einverleibung zu unterlaufen). Lipietz bleibt allerdings bei einführenden Erläuterungen nicht stehen; vor allem seine Diskussion eines "technologischen Auswegs aus der Krise «läßt sich als präzise Kontraposition zu den Ausführungen von Alfred Kleinknecht in PROKLA 57, aber auch zu der These eines neuen "politischen Regulierungsmodells« in Gestalt des CDU-Staats, wie sie Josef Esser und Joachim Hirsch in PROKLA 56 entwickelt haben, lesen.

Die nächste Ausgabe der PROKLA wird sich mit dem Komplex Weltwirtschaft - Abhängigkeit - Entwicklungsperspektiven beschäftigen.

\section{Die Redaktion}

\section{Errata}

Im Beitrag von Jörg Glomboreski/Michael Krüger (PROKLA 57) ist uns ein Versehen unterlaufen. Die Abbildung 1 auf S. 44 ist falsch herum übernommen worden. Abbildung 1 gibt einen dynamischen Prozeß in der von den Variablen $\beta$ und $\lambda$ aufgespannten Ebene wieder. Dabei wird $\beta$ auf der Ordinate (mit Pfeilrichtung nach oben!) und $\lambda$ auf der Abszisse abgetragen. Man erkennt dann sofort, daß das System dem Gleichgewichtspunkt $\left(\beta_{0}, \lambda_{0}\right)$ entgegenstrebt.

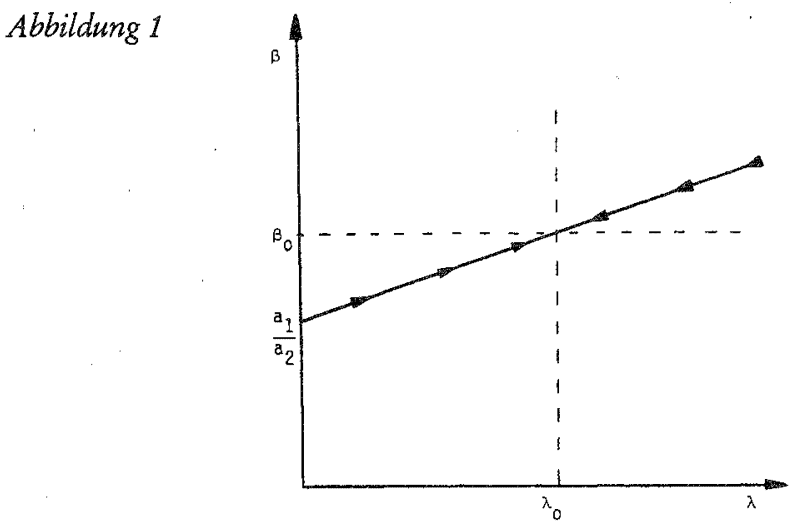

